\title{
DISTRIBUTION OF SPECIAL SEQUENCES MODULO A LARGE PRIME
}

\section{Z. GARAEV and KA-LAM KUEH}

\author{
Received 18 June 2002
}

\begin{abstract}
We study the sets $\left\{g^{x}-g^{y}(\bmod p): 1 \leq x, y \leq N\right\}$ and $\{x y: 1 \leq x, y \leq N\}$ where $p$ is a large prime number, $g$ is a primitive root, and $p^{2 / 3}<N<p$.

2000 Mathematics Subject Classification: 11A07.
\end{abstract}

1. Introduction. Let $p$ be a large prime number, $g$ a primitive root $(\bmod p)$, and $N$ a given positive integer, $N<p$. In a series of papers, the distribution of powers $g^{n}(\bmod p)$ has been investigated by [1, 2, 4, 5]. Vâjâitu and Zaharescu [5] considered the question of A. Odlyzko concerning the set of differences

$$
A:=\left\{g^{x}-g^{y}(\bmod p): 1 \leq x, y \leq N\right\} .
$$

As it was indicated in [5], A. Odlyzko asks for which values of $N$ the set $A$ contains all residue classes $(\bmod p)$. The conjecture is that one can take $N$ to be as small as $p^{1 / 2+\varepsilon}$, for any positive $\varepsilon$ and $p>c$ with some $c=c(\varepsilon)$. From the result of Rudnick and Zaharescu [4] it follows that in Odlyzko's problem one can take $N=c_{0} p^{3 / 4} \log p$ for some absolute constant $c_{0}$.

One of the main results of [5] is that for the exceptional set of Odlyzko's problem we have

$$
\#\{h(\bmod p): h \notin A\} \ll \frac{p^{3} \log p}{N^{3}} .
$$

It then follows that for $N>p^{2 / 3+\varepsilon}$ almost all the residues $(\bmod p)$ belong to $A$.

Denote

$$
B=\{x y(\bmod p): 1 \leq x, y \leq N\} .
$$

Vâjâitu and Zaharescu [5] put another problem similar to that of Odlyzko: for which values of $N$ can we be sure that the set $B$ contains all residue classes $(\bmod p)$ ? They conjectured that $N$ can be taken to be as small as $p^{1 / 2+\varepsilon}$ and 
observed that one can take $N=c_{1} p^{3 / 4} \log p$. This problem is also related to the pair correlation problem for sequences of the form $\alpha n^{2}(\bmod 1)$. For this account, see Rudnick et al. [3].

In this paper, using an elementary approach we slightly improve by a factor of $\log p$ estimate (1.2) and the estimate for $N$ in Odlyzko's problem and obtain estimate (1.2) with the set $B$ instead of $A$, see Theorems 1.1, 1.2, and 1.3.

THEOREM 1.1. For any prime number $p$, any primitive root $g(\bmod p)$, and $N=10 p^{3 / 4}$, the set A contains the complete residue system $(\bmod p)$.

THEOREM 1.2. For any prime number $p$, any primitive root $g(\bmod p)$, and any positive integer $N<p$,

$$
\#\{h(\bmod p): h \notin A\} \ll \frac{p^{3}}{N^{3}} .
$$

THEOREM 1.3. For any prime number $p$ and any positive integer $N<p$,

$$
\#\{h(\bmod p): h \notin B\} \ll \frac{p^{3} \log p}{N^{3}} .
$$

We require the following lemma (see [6, Exercise 14, page 92] and the solution in [6, page 142]) which will be used in the proof of Theorems 1.1 and 1.2 .

LEMMA 1.4. Let $m>1,(a, m)=1$. Then

$$
\left|\sum_{x=0}^{m-1} \sum_{y=0}^{m-1} v(x) \varrho(y) e^{2 \pi i(a x y / m)}\right| \leq \sqrt{m X Y},
$$

where $v(x), \varrho(y)$ are complex numbers and

$$
\sum_{x=0}^{m-1}|v(x)|^{2}=X, \quad \sum_{y=0}^{m-1}|\varrho(y)|^{2}=Y .
$$

2. Proof of Theorem 1.1. Note that $0 \in A$. Let $h$ be any integer, $h \neq 0(\bmod p)$, $N=10 p^{3 / 4}$, and denote $N_{1}=[N / 4]$. Our aim is to prove that $J>0$, where $J$ is the number of solutions in integers $x, y, z$, and $t$ of the congruence equation

$$
g^{x+z}-g^{y}-h g^{t} \equiv 0(\bmod p)
$$

subject to the condition

$$
N_{1}+1 \leq x, y, z \leq 2 N_{1}, \quad 1 \leq t \leq N_{1} .
$$

In order to prove it we write $J$ in terms of rational trigonometric sums:

$$
p J=\sum_{a=0}^{p-1} \sum_{x=N_{1}+1}^{2 N_{1}} \sum_{y=N_{1}+1}^{2 N_{1}} \sum_{z=N_{1}+1}^{2 N_{1}} \sum_{t=1}^{N_{1}} e^{2 \pi i\left(a\left(g^{x+z}-g^{y}-h g^{t}\right) / p\right)} .
$$


Picking up the term with $a=0$ and estimating other terms by their absolute values, we obtain

$$
\begin{aligned}
& p J \geq N_{1}^{4}-\sum_{a=1}^{p-1} \mid \sum_{x=N_{1}+1}^{2 N_{1}} \sum_{z=N_{1}+1}^{2 N_{1}} e^{2 \pi i\left(a g^{x} g^{z} / p\right)} \mid \\
& \times\left|\sum_{y=N_{1}+1}^{2 N_{1}} e^{2 \pi i\left(a g^{y} / p\right)}\right|\left|\sum_{t=1}^{N_{1}} e^{2 \pi i\left(a h g^{t} / p\right)}\right| .
\end{aligned}
$$

We will apply Lemma 1.4 to the double inner sum. To do that, we define $v(u)=\varrho(u)=1$ if $u \equiv g^{x}(\bmod p)$ for some $N_{1}+1 \leq x \leq 2 N_{1}$. For all other $u$, we put $v(u)=\varrho(u)=0$. Then Lemma 1.4 gives

$$
\left|\sum_{x=N_{1}+1}^{2 N_{1}} \sum_{z=N_{1}+1}^{2 N_{1}} e^{2 \pi i\left(a g^{x} g^{z} / p\right)}\right| \leq \sqrt{p N_{1}^{2}}
$$

Hence,

$$
p J \geq N_{1}^{4}-\sqrt{p N_{1}^{2}} \sum_{a=0}^{p-1}\left|\sum_{y=N_{1}+1}^{2 N_{1}} e^{2 \pi i\left(a g^{y} / p\right)}\right|\left|\sum_{t=1}^{N_{1}} e^{2 \pi i\left(a h g^{t} / p\right)}\right| .
$$

For the sum over $a$, we apply Cauchy inequality. Since $g$ is a primitive root, then

$$
\sum_{a=0}^{p-1}\left|\sum_{y=N_{1}+1}^{2 N_{1}} e^{2 \pi i\left(a g^{y} / p\right)}\right|^{2}=p N_{1}, \quad \sum_{a=0}^{p-1}\left|\sum_{t=1}^{N_{1}} e^{2 \pi i\left(a h g^{t} / p\right)}\right|^{2}=p N_{1} .
$$

Therefore, for each integer $h$,

$$
p J>N_{1}^{4}-p^{3 / 2} N_{1}^{2}
$$

and Theorem 1.1 follows in view of $N_{1}=[N / 4]$.

3. Proof of Theorem 1.2. Denote $\bar{A}=\{h(\bmod p): h \notin A\}, N_{1}=[N / 2]$, and let $|\bar{A}|$ denote the cardinality of $\bar{A}$. Then

$$
\sum_{h \in \bar{A}} \sum_{a=0}^{p-1} \sum_{x=1}^{N_{1}} \sum_{z=1}^{N_{1}} \sum_{y=1}^{N} e^{2 \pi i\left(a\left(g^{x+z}-g^{y}-h\right) / p\right)}=0 .
$$

Picking up the term with $a=0$, we obtain

$$
N_{1}^{2} N|\bar{A}| \leq \sum_{a=1}^{p-1}\left|\sum_{x=1}^{N_{1}} \sum_{z=1}^{N_{1}} e^{2 \pi i\left(a g^{x} g^{z} / p\right)}\right|\left|\sum_{y=1}^{N} e^{2 \pi i\left(a g^{y} / p\right)}\right|\left|\sum_{h \in \bar{A}} e^{2 \pi i(a h / p)}\right| .
$$


We will apply Lemma 1.4 to the double inner sum in the same way as we did in the proof of Theorem 1.1. We obtain

$$
\left|\sum_{x=1}^{N_{1}} \sum_{z=1}^{N_{1}} e^{2 \pi i\left(a g^{x} g^{z} / p\right)}\right| \leq \sqrt{p N_{1}^{2}} .
$$

Hence,

$$
N_{1}^{2} N|\bar{A}| \leq \sqrt{p N_{1}^{2}} \sum_{a=0}^{p-1}\left|\sum_{y=1}^{N} e^{2 \pi i\left(a g^{y} / p\right)}\right|\left|\sum_{h \in \bar{A}} e^{2 \pi i(a h / p)}\right| .
$$

In analogy with Section 2, we apply Cauchy inequality to the sum over $a$. Since

$$
\begin{aligned}
& \sum_{a=0}^{p-1}\left|\sum_{y=1}^{N} e^{2 \pi i\left(a g^{y} / p\right)}\right|^{2}=p N, \\
& \sum_{a=0}^{p-1}\left|\sum_{h \in \bar{A}} e^{2 \pi i(a h / p)}\right|^{2}=p|\bar{A}|,
\end{aligned}
$$

then

$$
N_{1}^{2} N|\bar{A}| \leq \sqrt{p N_{1}^{2} p N p|\bar{A}|} .
$$

Hence, from $N_{1}=[N / 2]$, we obtain

$$
|\bar{A}| \leq \frac{10 p^{3}}{N^{3}} .
$$

This proves Theorem 1.2.

4. Proof of Theorem 1.3. Using Gauss method of estimation of trigonometric sums, one can prove the validity of the following lemma.

LEMMA 4.1. Let $1 \leq N \leq p,(a, p)=1$. Then

$$
\left|\sum_{x=1}^{N} e^{2 \pi i\left(a x^{2} / p\right)}\right| \ll \sqrt{p \log p} .
$$

Indeed, if we denote by $|S|$ the value of the left-hand side, then

$$
|S|^{2}=\sum_{x=1}^{N} \sum_{y=1}^{N} e^{2 \pi i\left(a\left(y^{2}-x^{2}\right) / p\right)} \leq N+2\left|\sum_{1 \leq x<y \leq N} e^{2 \pi i\left(a\left(y^{2}-x^{2}\right) / p\right)}\right| .
$$

Substituting $y=x+t$ gives

$$
|S|^{2} \ll N+\left|\sum_{x=1}^{N-1} \sum_{t=1}^{N-x} e^{2 \pi i\left(a t^{2}+2 a t x / p\right)}\right| .
$$


Changing the order of summation, we obtain

$$
|S|^{2} \ll N+\sum_{t=1}^{N-1}\left|\sum_{x=1}^{N-t} e^{2 \pi i(2 a t x / p)}\right| \ll N+\sum_{t=1}^{p-1} \frac{1}{|\sin (\pi 2 a t / p)|}
$$

When $t$ runs through reduced residue system $(\bmod p)$ so does $2 a t$. Hence,

$$
|S|^{2} \ll N+\sum_{t=1}^{p-1} \frac{1}{|\sin (\pi t / p)|} \ll N+\sum_{t=1}^{(p-1) / 2} \frac{1}{t / p} \ll p \log p
$$

We now proceed to prove Theorem 1.3. Put $N_{1}=[N / 4]$ and denote by $B_{1}$ the set

$$
B_{1}=\left\{x^{2}-y^{2}(\bmod p), N_{1} \leq x \leq 2 N_{1}, 1 \leq y<N_{1}\right\}
$$

Since $B_{1} \subset B$, then $|\bar{B}| \leq\left|\bar{B}_{1}\right|$ where $\bar{B}$ and $\bar{B}_{1}$ denote the complement of $B$ and $B_{1}$ in the complete residue system $(\bmod p)$, accordingly. Now, as in the proof of Theorem 1.2, we have

$$
\sum_{h \in \overline{B_{1}}} \sum_{a=0}^{p-1} \sum_{x=N_{1}}^{2 N_{1}} \sum_{y=1}^{N_{1}-1} e^{2 \pi i\left(a\left(x^{2}-y^{2}-h\right) / p\right)}=0
$$

Then it follows that

$$
N^{2}\left|\overline{B_{1}}\right| \ll \sum_{a=1}^{p-1}\left|\sum_{x=N_{1}}^{2 N_{1}} e^{2 \pi i\left(a x^{2} / p\right)}\right|\left|\sum_{y=1}^{N_{1}-1} e^{2 \pi i\left(a y^{2} / p\right)}\right|\left|\sum_{h \in \overline{B_{1}}} e^{2 \pi i(a h / p)}\right| .
$$

Now, apply Lemma 4.1 for the sum over $x$ and then use Cauchy inequality as we did in the proof of Theorems 1.1 and 1.2. Then, we obtain

$$
N^{2}\left|\overline{B_{1}}\right| \ll \sqrt{p \log p} \sqrt{p N p\left|\overline{B_{1}}\right|}
$$

whence, we get

$$
\left|\overline{B_{1}}\right| \ll \frac{p^{3} \log p}{N^{3}}
$$

Now, Theorem 1.3 follows from $|\bar{B}| \leq\left|\overline{B_{1}}\right|$.

ACKNOWLedgment. This paper is supported by the NSC Grant 91-2115-M001-020. 


\section{REFERENCES}

[1] C. I. Cobeli, S. M. Gonek, and A. Zaharescu, On the distribution of small powers of a primitive root, J. Number Theory 88 (2001), no. 1, 49-58.

[2] H. L. Montgomery, Distribution of small powers of a primitive root, Advances in Number Theory (Kingston, Ontario, 1991), Oxford Sci. Publ., Oxford University Press, New York, 1993, pp. 137-149.

[3] Z. Rudnick, P. Sarnak, and A. Zaharescu, The distribution of spacings between the fractional parts of $n^{2} \alpha$, Invent. Math. 145 (2001), no. 1, 37-57.

[4] Z. Rudnick and A. Zaharescu, The distribution of spacings between small powers of a primitive root, Israel J. Math. 120 (2000), 271-287.

[5] M. Vâjâitu and A. Zaharescu, Differences between powers of a primitive root, Int. J. Math. Math. Sci. 29 (2002), no. 6, 325-331.

[6] I. M. Vinogradov, An Introduction to the Theory of Numbers, Pergamon Press, London, 1955.

M. Z. Garaev: Institute of Mathematics, Academia Sinica, Nankang, Taipei 11529, Taiwan

E-mail address: garaev@math.sinica.edu.tw

Ka-Lam Kueh: Institute of Mathematics, Academia Sinica, Nankang, Taipei 11529, Taiwan

E-mail address: mak1k@ccvax.sinica.edu.tw 


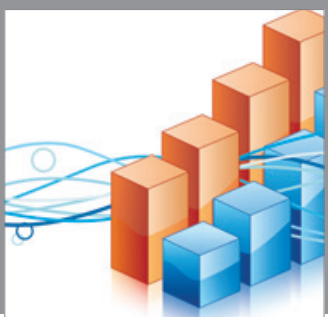

Advances in

Operations Research

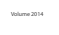

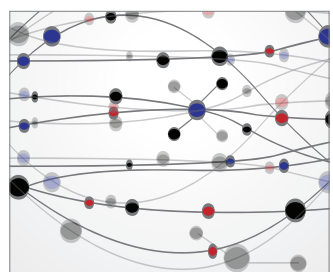

\section{The Scientific} World Journal
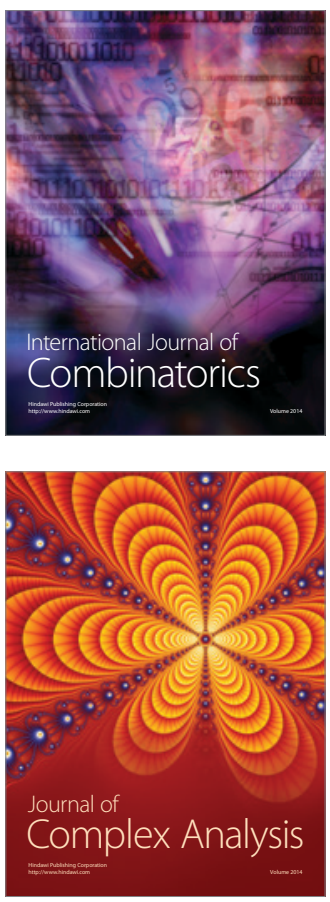

International Journal of

Mathematics and

Mathematical

Sciences
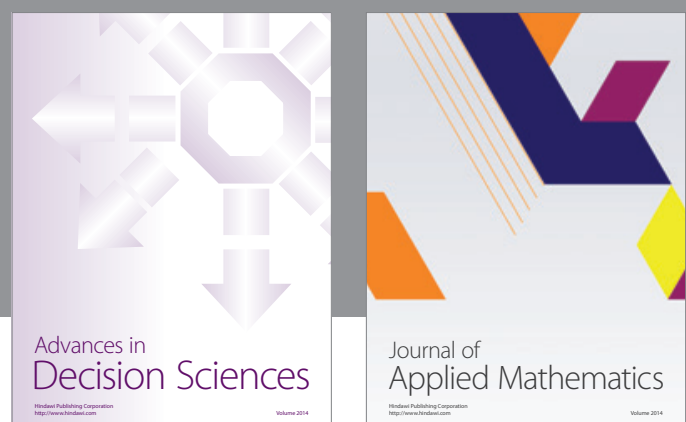

Journal of

Applied Mathematics
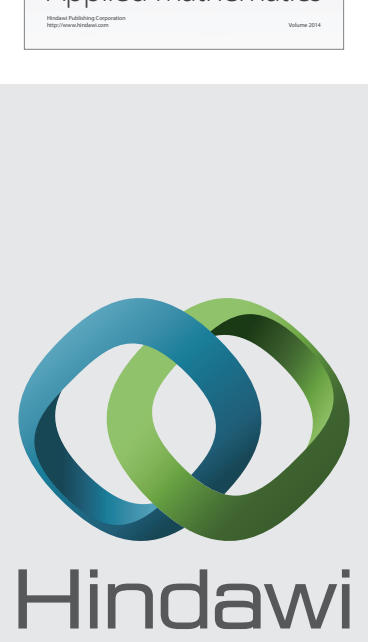

Submit your manuscripts at http://www.hindawi.com
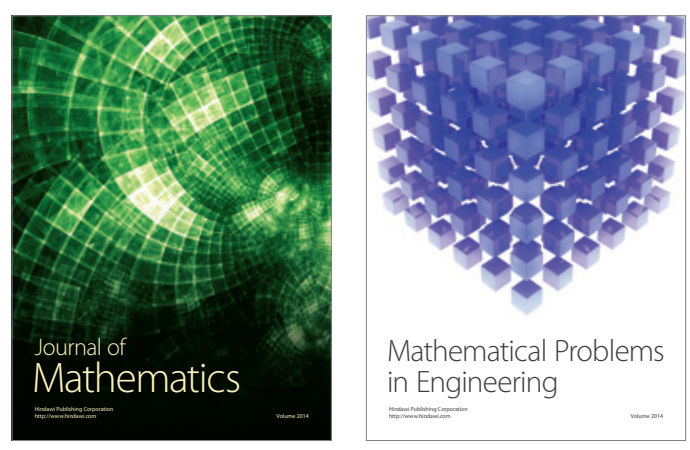

Mathematical Problems in Engineering
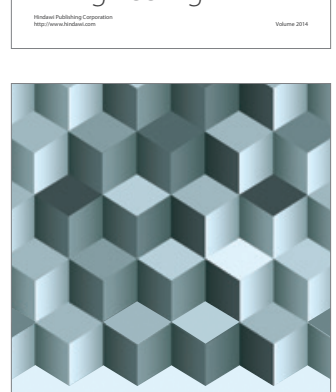

Journal of

Function Spaces
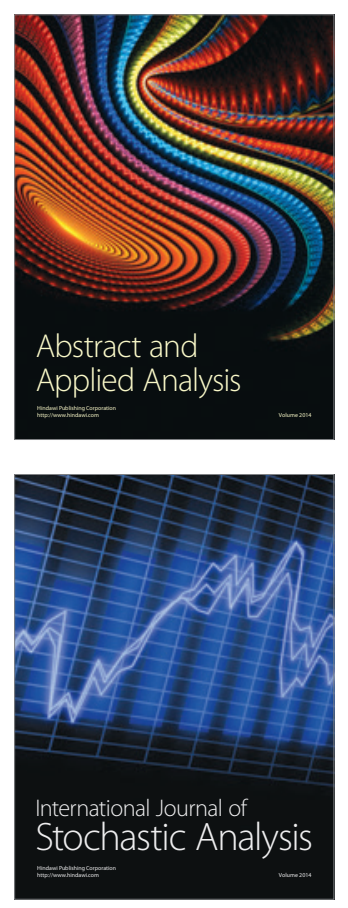

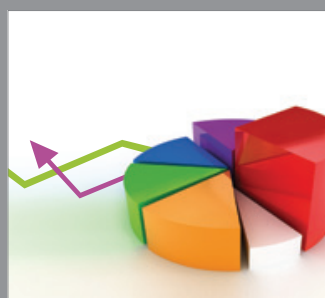

ournal of

Probability and Statistics

Promensencen
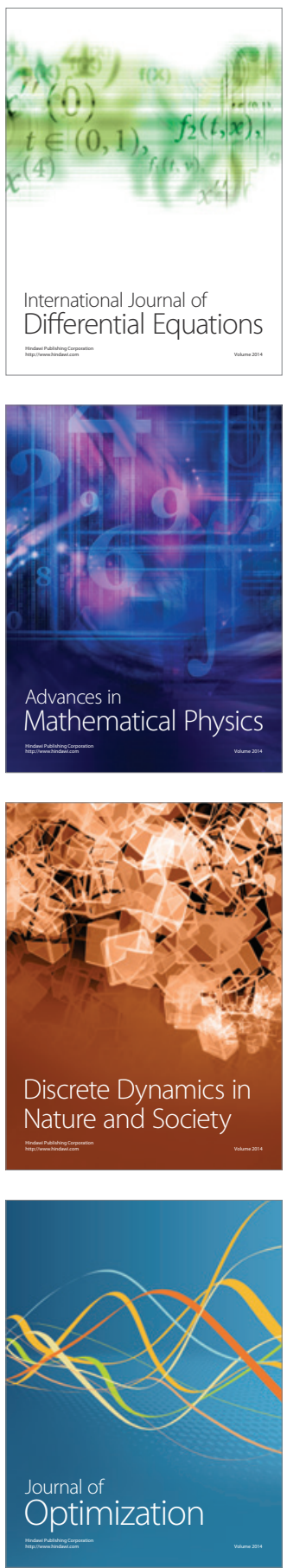